\title{
On the arithmetic of genus two fibrations
}

\author{
by \\ Mustafa D. Kaba and Hurşit Önsiper (Ankara)
}

In this paper, we are concerned with the arithmetic of surfaces which have irregularity $q=2$, and which admit nonsmooth genus two fibrations over elliptic curves, defined over a number field $K$. For such a surface $S$, the geometric properties one needs for arithmetic purposes are quite well known. Employing these properties to compare $S$ and the Albanese variety $A$ of $S$, we study the following special cases of a vast set of conjectures stated by Tate ([5, p. 97], [6, p. 72]) for any smooth projective variety defined over a field which is finitely generated over the prime field:

$$
\begin{array}{ll}
T^{1}(S): & \mathrm{NS}(S) \otimes \mathbb{Q}_{l}=\left(H_{\mathrm{et}}^{2}\left(\bar{S}, \mathbb{Q}_{l}\right)(1)\right)^{\operatorname{Gal}(\bar{K} / K)}, \\
L_{2}(S): & \varrho(S)=-\operatorname{ord}_{s=2}\left(L_{S, 2}(s)\right),
\end{array}
$$

where $L_{S, 2}(s)$ is the factor of the zeta function $\zeta_{S}(s)=\prod_{i=0}^{4} L_{S, i}(s)^{(-1)^{i}}$ arising from the second cohomology.

The techniques we employ are quite standard computations in cohomology. First working over the algebraic closure $\bar{K}$ of $K$ we recall the essentials of the geometry of $\bar{S}=S \otimes \bar{K}$ and we calculate the rank $\varrho(\bar{S})($ resp. $\varrho(\bar{A})$ ) of the Néron-Severi group $\operatorname{NS}(\bar{S})$ (resp. $\operatorname{NS}(\bar{A})$ ) of $\bar{S}$ (resp. the Albanese variety $\bar{A}$ of $\bar{S}$ ). These calculations yield the following result.

Proposition 1. $\varrho(\bar{S})=\varrho(\bar{A})+N_{\mathrm{c}}$, where $N_{\mathrm{c}}$ is the number of curves contracted by the Albanese map $\alpha: \bar{S} \rightarrow \bar{A}$.

For the precise statement of the result on the conjecture $L_{2}(S)$ we let $E^{\prime}$ denote the elliptic curve which is the subcover of the smooth fibers of the fibration $\Psi: S \rightarrow E$ over the elliptic curve $E$. We note that $E^{\prime}$ is the $K(E) / K$-image of the jacobian $\left(\mathcal{J}_{S / E}\right)_{\eta}$ of the generic fiber of $\Psi$. As a consequence of self duality of the jacobian, $E^{\prime}$ is also the $K(E) / K$-trace of the relative jacobian $\left(\mathcal{J}_{S / E}\right)$.

2000 Mathematics Subject Classification: 14D06, 14G10.

Key words and phrases: fibrations, arithmetic. 
Proposition 2. Let $S$ be a smooth projective surface with irregularity $q(S)=2$ defined over a number field $K$ and let $A$ be the Albanese variety of $S$. Suppose that $S$ admits a fibration (defined over $K) \Psi: S \rightarrow E$ over an elliptic curve $E$ and let $E^{\prime}$ be the elliptic subcover of the smooth fibers of $\Psi$. Then $L_{2}(S)$ is true if and only if $L_{2}(A)$ is true. In particular, $L_{2}(S)$ holds if $E$ and $E^{\prime}$ have complex multiplication.

Proposition 3. NS $(S) \otimes \mathbb{Q}_{l}=\left(H_{\text {et }}^{2}\left(\bar{S}, \mathbb{Q}_{l}\right)(1)\right)^{\operatorname{Gal}(\bar{K} / K)}$.

Throughout the paper we will use the following basic notation and definitions.

For a curve $C, k(C)$ denotes the function field of $C$ and $J_{C}$ is the jacobian variety of $C$.

For a surface $S$,

- $\chi(S)$ is the holomorphic Euler characteristic.

- $K(S), w(S)$ denote the canonical divisor and the canonical bundle.

- $c_{1}(S), c_{2}(S)$ are the first and second Chern classes of $S$.

- $\mathrm{NS}(S)$ denotes the Néron-Severi group.

- $\varrho(S)$ denotes the Picard number.

For a fibration $S \rightarrow C, K_{S / C}$ denotes the relative canonical divisor.

Definition 1. For any integer $d \geq 2, X(d)$ is the complete modular curve obtained from the quotient of the upper half plane by the modular group $\Gamma(d)$.

Definition 2. For a locally nontrivial minimal fibration $S \rightarrow C$ with fiber genus $g$, the slope $\lambda$ is defined by

$$
\lambda=K_{S / C}^{2} /(\chi(S)-(g-1)(g(C)-1)) .
$$

Clearly for the fibrations considered in this paper we have $K^{2}(S)=$ $\lambda \chi(S)$.

Definition 3. For a fibration $S \rightarrow C$, the jacobian fibration $\mathcal{J}_{S / C}$ is the connected component $\mathrm{Pic}_{S / C}^{0}$ of the Picard scheme $\mathrm{Pic}_{S / C}$.

Let $S$ be a projective smooth surface admitting a genus two fibration $\Psi: S \rightarrow E$ with $q(S)=2$ over an elliptic curve. We assume that all this structure is defined over a number field $K$.

In case the fibration is smooth, it is isotrivial with monodromy group isomorphic to $\mathbb{Z}_{2}$ and hence the arithmetic of these fibrations is easy to work out. The interesting case is that of nonsmooth fibrations. For such fibrations we know that the slope (Definition 2) is given by $\lambda=7-6 / d$ for some $d=2,3,4,5,6$ and that $S$ is a minimal surface of general type. Moreover, the fibration is semi-stable and nonisotrivial if $d \geq 3$ ([7, Corollaire, p. 47]). In fact, for any given elliptic curve $E^{\prime}$ and $d \geq 3$, we have a semi-stable genus 
two fibration $\Pi: S\left(E^{\prime}, d\right) \rightarrow X(d)$ on the modular curve $X(d)$ (Definition 1) such that any fibration $S \rightarrow E$ with $E^{\prime}$ as the elliptic subcover of degree $d$ of the smooth fibers of $S \rightarrow E$, is obtained as the minimal desingularization of the pull-back $f^{*}\left(S\left(E^{\prime}, d\right)\right)$ by a nontrivial morphism $f: E \rightarrow X(d)$ ([7, Théorème 3.10], cf. [1, Corollary 1.2]).

For the existence of such surfaces we have

Lemma 1. Let $E, E^{\prime}$ be elliptic curves defined over a number field $K$ which contains the cyclotomic field $\mathbb{Q}\left(\zeta_{d}\right), 3 \leq d \leq 6$. For $d=6$, we assume that $E \sim X(6)$. Then there exists a genus two fibration $\Psi: S \rightarrow E$ defined over a finite algebraic extension of $K$ with $q(S)=2$ such that $E^{\prime}$ is a subcover of degree $d$ of the smooth fibers.

Proof. With the given hypothesis we have a surjective map $E \rightarrow X(d)$. Therefore we have a smooth genus two fibration $S_{U} \rightarrow U$ over an open subscheme $U \subset E$ ([1, Corollary 1.2]) with $E^{\prime}$ as a subcover of degree $d$ of the fibers, defined over $K$. Since this family is projective over $U$, we can imbed $S_{U}$ in a suitable projective space $\mathbb{P}_{S}^{n}$ and take its closure $S^{\prime}$ in $\mathbb{P}_{S}^{n}$. Resolving the singularities of $S^{\prime}$ we obtain $S$ as required.

In analyzing the geometry of the given surface, we work over the algebraic closure $\bar{K}$ of $K$, but we will permit a conflict with our notation in the introduction and a certain abuse of notation by using the same symbols for all objects defined over $K$ and those obtained by the base extension $\operatorname{Spec}(\bar{K}) \rightarrow \operatorname{Spec}(K)$.

For the main results on the geometric structure of these surfaces we will refer to [7]: the surface $S\left(E^{\prime}, d\right)$ has two types of singular fibers ([7, Lemme 3.11, Théorème 3.16]):

- Type $I$ : Fibers over the cusps of the modular curve $X(d)$ are curves with a node such that the normalization is an elliptic curve.

- Type II: Singular fibers over the ordinary points of $X(d)$ consist of two elliptic curves meeting transversally at a single point.

Therefore, on $S$, curves of geometric genus 0 all come from the resolution of the singularities of the surface $f^{*}\left(S\left(E^{\prime}, d\right)\right)$. The latter has singular points if and only if $f$ has branch points over which singular fibers of $S\left(E^{\prime}, d\right) \rightarrow X(d)$ lie. In such a case, if $f$ has ramification index $k+1$ at $p$ on $E$, then the fiber of $f^{*}\left(S\left(E^{\prime}, d\right)\right) \rightarrow E$ over $p$ has a singular point of type $A_{k}$.

In the rest of the paper, we will say that a singular fiber $F$ on $S$ is of Type I (resp. Type II) if $F$ is mapped onto a fiber of Type I (resp. Type II) by the natural morphism $S \rightarrow S\left(E^{\prime}, d\right)$. The cycle configuration of a singular fiber which contains an $A_{k}$-chain is as follows:

- Type I: a closed cycle consisting of a smooth elliptic curve and $k$ smooth rational curves, 
- Type II: two disjoint elliptic curves, one of which intersects the first component and the other intersects the last component of a chain of $k$ smooth rational curves.

The Albanese map $\alpha: S \rightarrow A$ is generically finite of degree $d ; \alpha$ contracts precisely one elliptic component from each fiber of Type II and all the $A_{k^{-}}$ chains on $S$. We have

Proposition 1. $\varrho(S)=\varrho(A)+N_{\mathrm{c}}$, where $N_{\mathrm{c}}$ is the number of irreducible curves contracted by $\alpha$.

Proof. Replacing $A$ by the image $\alpha(Y)$ of the Albanese map $\alpha: Y \rightarrow A$, we will prove the result for any genus two fibration $Y \rightarrow C$ with $q(Y)=$ $g(C)+1 \geq 2$.

Clearly, we may assume that $Y \rightarrow C$ has a section $s: C \rightarrow Y$.

Let $\phi: \operatorname{Alb}_{Y} \rightarrow J_{C}$ be the map induced by the universal mapping property of $\operatorname{Alb}_{Y}$. Then $\phi$ is an elliptic fibration with fiber $E^{\prime}=$ the $K(C) / K$ image of $\mathcal{J}_{Y / C}$ (Definition 3). Hence the image $\alpha(Y) \subset \mathrm{Alb}_{Y}$ is an elliptic curve over the image $i(C)$ of $C$ in $J_{C}$ with zero section given by $\alpha \circ s$. Therefore, by the universal mapping property of $\mathcal{J}_{Y / C}$, we obtain a $C$-morphism $\psi: \mathcal{J}_{Y / C} \rightarrow \alpha(Y)$. By [3, Theorem 1] we have $\varrho(Y)=$ $\varrho\left(\mathcal{J}_{\eta}(K(C)) / E^{\prime}(K)\right)+N_{\mathrm{c}}+2$ where $\mathcal{J}_{\eta}$ is the generic fiber of the jacobian fibration $\mathcal{J}_{Y / C}$. On the other hand, since $E^{\prime}$ is the $K(C) / K$-image of $\mathcal{J}_{Y / C}$, $p_{1}, p_{2} \in \mathcal{J}_{\eta}(K(C))$ are equivalent $\bmod E^{\prime}(K)$ if and only if $\psi\left(p_{1}\right)-\psi\left(p_{2}\right) \in$ $E^{\prime}(K) \subset \alpha(Y)_{\eta}(K(C))$. But $\alpha(Y)$ being an elliptic surface with no multiple fibers, we have $\varrho(\alpha(Y))=\varrho\left(\alpha(Y)_{\eta}(K(C))+2\right.$. The proposition follows.

Since $A \sim E \times E^{\prime}$ we have $\varrho(A)=\varrho\left(E \times E^{\prime}\right)$ and thus we obtain

Corollary 1. For $S$ as in Proposition 1 we have

$$
\varrho(S)= \begin{cases}4+N_{\mathrm{c}} & \text { if } E \sim E^{\prime} \text { and } E \text { has } C M, \\ 3+N_{\mathrm{c}} & \text { if } E \sim E^{\prime} \text { and } E \text { does not have } C M, \\ 2+N_{\mathrm{c}} & \text { if } E \nsim E^{\prime} .\end{cases}
$$

ExAmple. For $d=6$, the modular curve $X(6)$ is an elliptic curve with $j(X(6))=0$, hence is of CM-type. Since the base curve $E$ is isogeneous to $X(6)$, it is also of CM-type. Furthermore, $S$ has precisely $c_{2}(S)=36 n$ singular fibers where $n=\operatorname{deg}(f), f: E \rightarrow X(6)$ is the morphism in [7, Théorème 3.10]. Precisely $24 n$ of these fibers are of Type II and hence $N_{\mathrm{c}}=$ $24 n$. Thus we see that $\varrho(S)=4+2 c_{2}(S) / 3\left(\right.$ resp. $\left.2+2 c_{2}(S) / 3\right)$ if $E \sim E^{\prime}$ (resp. $E \not E^{\prime}$ ). In fact, one has $h^{1,1}(S)=4+2 c_{2}(S) / 3$ and therefore $2+2 c_{2}(S) / 3 \leq \varrho(S) \leq 4+2 c_{2}(S) / 3$. Thus in this case $\varrho(S)$ attains both bounds.

Next we address Tate's conjectures. After taking a finite extension of $K$ if necessary, we assume that all singular fibers of the given fibration $\Psi: S \rightarrow E$ 
are defined over $K$ and that in the normalization of such a fiber the double point splits into two $K$-rational points. Furthermore, we require that the Néron-Severi group $\operatorname{NS}(\bar{S})$ of $\bar{S}=S \otimes_{K} \bar{K}$ is generated by the classes of divisors defined over $K$.

Proposition 2 is obtained from Lemma 2 and Corollary 2 given below. In the proof of Lemma 2 we will compare the standard exact sequence of etale cohomology with compact supports for the open subscheme $U=S-Y$ of $S$ where $Y$ is the union of the singular fibers of $\Psi$, and the exact sequence for $U^{\prime}=\alpha(U), Y^{\prime}=\alpha(Y)$ :

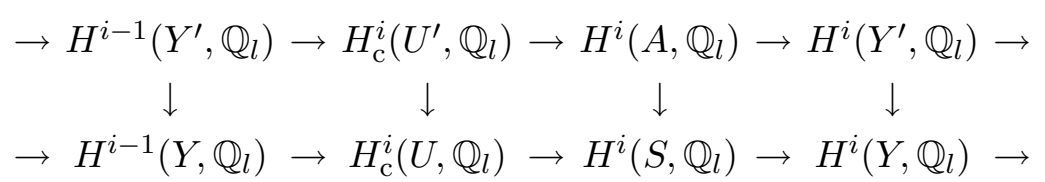

Using the geometry of singular fibers we see that

- $h^{1}(Y)=h^{1}\left(Y^{\prime}\right)+\delta_{\mathrm{I}}$ where $\delta_{\mathrm{I}}$ is the number of fibers of Type I,

- $h^{2}(Y)=h^{2}\left(Y^{\prime}\right)+N_{\mathrm{c}}$.

Therefore, comparing the etale Euler characteristics $\chi(S)=c_{2}(S)=\delta_{\mathrm{I}}+N_{\mathrm{c}}$, $\chi(A)=0$ we obtain $h_{\mathrm{c}}^{i}\left(U^{\prime}\right)=h_{\mathrm{c}}^{i}(U)$ for all $i$.

Lemma 2. $L_{2}(S)$ is true if and only if $L_{2}(A)$ is true.

Proof. We know that $\alpha^{*}: H^{i}\left(A, \mathbb{Q}_{l}\right) \rightarrow H^{i}\left(S, \mathbb{Q}_{l}\right)$ is an isomorphism for $i \neq 2$ and is an injection for $i=2$. Hence $H^{2}\left(S, \mathbb{Q}_{l}\right)=\alpha^{*}\left(H^{2}\left(A, \mathbb{Q}_{l}\right)\right) \oplus V$ where $\operatorname{dim}(V)=c_{2}(S)=$ number of double points on the singular fibers.

Since $\alpha$ is defined over $K$, when reduced $\bmod \wp$ for a good prime $\wp \subset \mathcal{O}_{K}$ the induced maps respect the action of Frobenius $F$ on the cohomology groups. Therefore we see that

$$
\begin{aligned}
L_{S_{\wp}, 2}(s) & =\operatorname{det}\left(1-t F \mid H^{2}\left(S_{\wp}\right)\right)^{-1}=\operatorname{det}\left(1-t F \mid H^{2}\left(A_{\wp}\right)\right)^{-1} \operatorname{det}\left(1-t F \mid V_{\wp}\right)^{-1} \\
& =L_{A_{\wp}}(s) \operatorname{det}\left(1-t F \mid V_{\wp}\right)^{-1} .
\end{aligned}
$$

Taking the product over all $\wp$, we obtain $L_{S, 2}(s)=L_{A, 2}(s) \prod \operatorname{det}\left(1-t F \mid V_{\wp}\right)^{-1}$ up to finitely many factors due to bad primes.

To compute $\operatorname{det}\left(1-t F \mid V_{\wp}\right)$, we compare $\zeta_{S_{\wp}}(s)=\zeta_{U_{\wp}}(s) \zeta_{Y_{\wp}}(s)$ and $\zeta_{A_{\wp}}(s)=\zeta_{U_{\wp}^{\prime}}(s) \zeta_{Y_{\wp}^{\prime}}(s)$ using the diagram preceding the lemma (reduced $\bmod \wp)$. We find that the factor $\mathcal{F}_{\wp}$ of $\operatorname{det}\left(1-t F \mid V_{\wp}\right)$ which contributes to the difference $\operatorname{ord}_{s=2}\left(L_{S, 2}(s)\right)-\operatorname{ord}_{s=2}\left(L_{A, 2}(s)\right)$ is of the form

$$
\mathcal{F}_{\wp}=\prod \frac{\operatorname{det}\left(1-t F \mid H^{2}\left(\left(Y_{j}\right)_{\wp}\right)\right)}{\operatorname{det}\left(1-t F \mid H^{2}\left(Y_{\wp}^{\prime}\right)\right)}
$$

where the product is taken over the singular fibers. 
For a singular fiber $Y_{j}$ containing an $A_{k}$-cycle we have

$$
\frac{\operatorname{det}\left(1-t F \mid H^{2}\left(\left(Y_{j}\right)_{\wp}\right)\right)}{\operatorname{det}\left(1-t F \mid H^{2}\left(Y_{\wp}^{\prime}\right)\right)}= \begin{cases}(1-t N(\wp))^{k-1} & \text { if } Y_{j} \text { is of Type I } \\ (1-t N(\wp))^{k} & \text { if } Y_{j} \text { is of Type II. }\end{cases}
$$

Hence for each $Y_{j}, \prod_{\wp} \mathcal{F}_{\wp}=\zeta_{K}(s-1)^{-r}$ where $r=k-1$ (resp. $k$ ) if $Y_{j}$ is of Type I (resp. Type II). As this number $r$ is precisely the number of components in $Y_{j}$ contracted by $\alpha$, we see that

$$
\operatorname{ord}_{s=2} L_{S, 2}(s)=\operatorname{ord}_{s=2} L_{A, 2}(s)-N_{\mathrm{c}} .
$$

Combining with Proposition 1, we obtain Lemma 2.

Corollary 2. $L_{2}(S)$ holds if $E$ and $E^{\prime}$ have complex multiplication.

Proof. We need to verify that $\operatorname{ord}_{s=2} L_{A, 2}(s)=-2$ (resp. -4) if $E \nsim E^{\prime}$ (resp. $E \sim E^{\prime}$ ).

After reducing mod $\wp$ at a good prime of reduction for the fibration, we let $\pi_{i}, \bar{\pi}_{i}$ be the eigenvalues of Frobenius acting on $H^{1}\left(E_{\wp}, \mathbb{Q}_{l}\right)$ for $i=1$ and on $H^{1}\left(E_{\wp}^{\prime}, \mathbb{Q}_{l}\right)$ for $i=2$. Then on the component $H^{1}\left(E_{\wp}, \mathbb{Q}_{l}\right) \otimes H^{1}\left(E_{\wp}^{\prime}, \mathbb{Q}_{l}\right)$ of the Künneth decomposition for $H^{2}\left(A_{\wp}, \mathbb{Q}_{l}\right)$, the eigenvalues of Frobenius are $\pi_{1} \pi_{2}, \pi_{1} \bar{\pi}_{2}$ and their complex conjugates. On the factors $H^{2}\left(E_{\wp}\right) \otimes H^{0}\left(E_{\wp}^{\prime}\right)$ and $H^{0}\left(E_{\wp}\right) \otimes H^{2}\left(E_{\wp}^{\prime}\right)$ the eigenvalues are simply $N(\wp)$.

Therefore letting $\chi_{1}$ (resp. $\chi_{2}$ ) be the Hecke character for $E$ (resp. $E^{\prime}$ ) and observing that $\chi_{1}=\chi_{2}=\chi$ if and only if $E \sim E^{\prime}$, we see that up to finitely many factors due to bad primes, $L_{A, 2}(s)$ is of the form

$$
\zeta_{K}(s-1)^{4} L\left(s-1, \chi^{2}\right) L\left(s-1, \bar{\chi}^{2}\right)
$$

or

$$
\zeta_{K}(s-1)^{2} L\left(s-1, \chi_{1} \chi_{2}\right) L\left(s-1, \chi_{1} \bar{\chi}_{2}\right) L\left(s-1, \bar{\chi}_{1} \chi_{2}\right) L\left(s-1, \bar{\chi}_{1} \bar{\chi}_{2}\right)
$$

according as $E \sim E^{\prime}$ or not. Since none of the $L$-series occurring in these products vanishes at $s=2$, the conclusion follows.

REMARK. The computation in the proof of Corollary 2 is borrowed from the proof of [4, Theorem 6]. When $E \sim E^{\prime}$, the corollary is a special case of a much more general result in [2].

$$
\text { Proposition 3. NS }(S) \otimes \mathbb{Q}_{l}=\left(H_{\mathrm{et}}^{2}\left(\bar{S}, \mathbb{Q}_{l}\right)(1)\right)^{\mathrm{Gal}(\bar{K} / K)} .
$$

Proof. In the diagram preceding Lemma 2, all arrows respect the action of $G=\operatorname{Gal}(\bar{K} / K)$. We twist the sequences and denote by $h^{i}(Z)^{G}$ the dimension of the cohomology group $H^{i}\left(Z, \mathbb{Q}_{l}\right)^{G}$. We compute to find

$$
\begin{aligned}
& h^{2}(S)(1)^{G}=h_{\mathrm{c}}^{2}(U)(1)^{G}+h^{2}(Y)(1)^{G}-h^{1}(Y)(1)^{G}, \\
& h^{2}(A)(1)^{G}=h_{\mathrm{c}}^{2}\left(U^{\prime}\right)(1)^{G}+h^{2}\left(Y^{\prime}\right)(1)^{G}-h^{1}\left(Y^{\prime}\right)(1)^{G},
\end{aligned}
$$

where we have $h^{2}(Y)(1)^{G}-h^{2}\left(Y^{\prime}\right)(1)^{G}=N_{\mathrm{c}}, h^{1}(Y)(1)^{G}=h^{1}\left(Y^{\prime}\right)(1)^{G}$ and 
$h_{\mathrm{c}}^{2}(U)(1)^{G}=h_{\mathrm{c}}^{2}\left(U^{\prime}\right)(1)^{G}$. Since $T^{1}(A)$ holds, combining these observations with Proposition 1 we obtain the assertion.

Acknowledgements. The first author was supported by TUBITAK.

\section{References}

[1] E. Kani, Hurwitz spaces of genus 2 covers of an elliptic curve, Collect. Math. 54 (2003), 1-51.

[2] H. Pohlmann, Algebraic cycles on abelian varieties of complex multiplication type, Ann. of Math. 88 (1968), 161-180.

[3] T. Shioda, Mordell-Weil lattices for higher genus fibration over a curve, in: New Trends in Algebraic Geometry (Warwick, 1996), London Math. Soc. Lecture Note Ser. 264, Cambridge Univ. Press, 1999, 359-373.

[4] T. Shioda and H. Inose, On singular K3 surfaces, in: Complex Analysis and Algebraic Geometry, Iwanami Shoten, 1977, 119-136.

[5] J. Tate, Algebraic cycles and poles of zeta functions, in: Arithmetical Algebraic Geometry, Harper \& Row, 1964, 93-110.

[6] - Conjectures on algebraic cycles in l-adic cohomology, in: Motives, Proc. Sympos. Pure Appl. Math. 55, Part 1, Amer. Math. Soc., 1994, 71-83.

[7] G. Xiao, Surfaces Fibrées en Courbes de Genre Deux, Lecture Notes in Math. 1137, Springer, 1985.

Department of Mathematics

Middle East Technical University

06531 Ankara, Turkey

E-mail: e124469@metu.edu.tr

hursit@metu.edu.tr

Received on 5.5.2006

and in revised form on 12.3.2007 